\title{
Extended Criteria for Vitrectomy and Fluid/Silicone Oil Exchange
}

\author{
N. WILSON-HOLT and P. K. LEAVER \\ London
}

\begin{abstract}
Summary
The results of vitrectomy combined with fluid/silicone oil exchange at six months after surgery in 39 eyes with complex retinal detachments without (initially) severe proliferative vitreoretinopathy, proliferative diabetic retinopathy or giant retinal tears, are reported. Eyes with detachments as a result of macular holes, large multiple or posterior breaks, and eyes which had undergone previous, unsuccessful vitreoretinal surgery are included. In $90 \%$ the retina remained reattached, with functional improvement in $\mathbf{7 2} \%$. These findings, together with the relatively low incidence of complications observed, lead us to recommend this technique in the management of selected detachments falling outside the parameters within which its use is generally accepted.
\end{abstract}

The combination of vitrectomy and fluid-silicone oil exchange is now well established in the successful management of complicated retinal detachments, typically those with proliferative vitreoretinopathy (PVR), advanced proliferative diabetic retinopathy (PDR) and giant retinal tears $(\mathrm{GRT}) .^{1-6}$

This method is sometimes useful, however, in other circumstances where the special properties of silicone oil improve the prospects of achieving long term retinal reattachment. A consecutive series of such cases, not complicated by PDR, GRT or, initially, by PVR grades $C$ or $D$, is reported and the results after six months reviewed in order to assess the value of this method in their management.

\section{Materials and Methods}

Between October 1985 and October 1988, 269 cases of complicated retinal detachment under the care of one surgeon (PKL) at Moorfields Eye Hospital, were managed by vitrectomy and fluid/silicone-oil exchange.
In 230 eyes vitrectomy and fluid/silicone-oil exchange was undertaken for retinal detachment associated with PVR, PDR and GRT. Thirty-nine eyes did not meet these criteria and could be divided into four groups:

Group 1 (Macular Holes) $(n=10)$ Eight eyes had retinal detachments associated solely with macular breaks, seven in highly myopic eyes, and one in an emmetropic eye which had sustained severe blunt trauma. In two eyes, macular holes were associated with large peripheral breaks. Six eyes had undergone previous fluid/gas exchange, with failure of retinal reattachment, due to epiretinal membrane formation.

The remaining four eyes had epimacular membrane associated with the macular hole at presentation.

Group 2 (Large Multiple or Posterior Breaks) $(n=13)$ Four eyes had retinal detachments associated with large superior breaks and three eyes had large inferior breaks; six eyes 
had multiple posterior breaks. Two patients had Stickler's syndrome, and in one eye the breaks occurred in necrotic retina following resolved acute retinal necrosis. Two eyes had recently sustained penetrating trauma. In all instances it was felt that the eye was at substantial risk of developing PVR.

Group 3 (Recurrent or Persistent Retinal Detachment) $(\mathrm{n}=10)$ In 10 eyes which had undergone retinal reattachment surgery without vitrectomy and in which the retinal detachment persisted with open breaks, it was felt that the retina was at high risk of developing PVR.

The number of previous procedures ranged from one to four with a mean of 2.3. In six of these eyes drainage of subretinal fluid had been performed and in three intraocular gas had been injected. In one eye there was vitreoretinal incarceration related to external drainage of subretinal fluid, in one the retina remained detached in association with extensive choroidal effusions precluding the identification of retinal breaks prior to reoperation, and in one, retinal detachment persisted in the presence of endophthalmitis after an unsuccessful buckling procedure. In all cases the macula had been detached for at least one month.

Group 4 (Retinal Detachments Associated with Vitrectomy and Fluid/Gas Exchange) $(n=6)$ In six eyes retinal detachment occurred, persisted or recurred following vitrectomy and fluid/gas exchange. In five of these six eyes, vitrectomy and fluid/gas exchange had been undertaken for retinal detachments associated with multiple large breaks, three superior and two inferior. In one case retinal detachment was associated with an entry site break following vitrectomy for vitreous haemorrhage.

\section{Surgical Method}

Closed intraocular microsurgery, using a multiport system with 20 gauge instrumentation, was used in all cases. In three eyes lensectomy was performed at the time of surgery. Subretinal fluid was drained either internally (31 eyes) with a modified Charles flute needle or externally (eight eyes) with a 20 gauge needle under indirect ophthalmoscopic con- trol, and the intraocular fluid exchanged for silicone oil. Retinal breaks were treated either with transcleral cryotherapy (30 eyes) or endolaser photocoagulation (nine eyes, four of which had macular holes). Superior breaks were buckled in six of ten eyes, whilst inferior breaks were always buckled. Six out of 39 eyes were encircled at the time of fluid/ silicone oil exchange, using a number 40 silicone rubber band.

An inferior iridectomy (Ando) was undertaken to prevent pupil block by the oil globule whenever silicone oil was injected into an aphakic eye or when cataract extraction was performed in an oil-containing eye.

\section{Postoperative Management}

Patients were nursed in the face down position as soon as possible after surgery, both to ensure that aqueous rapidly filled the anterior chamber in front of the silicone oil globule and to achieve effective tamponade of retinal break(s). After early mobilisation, they were instructed not to lie in the supine position because the presence of a patent iridectomy, either at the 12 o'clock or 6 o'clock position, cannot always prevent pupil block by silicone oil if the supine position is assumed, while effective internal tamponade of retinal breaks may no longer be maintained.

\section{Reoperations}

In four eyes further reattachment procedures were undertaken within one month of the initial silicone oil exchange because the original tear(s) reopened or new tears developed. In one eye an epimacular membrane was peeled, and in two cases a drainage tube was inserted, because raised intraocular pressure could not be controlled satisfactorily with medical treatment. In six phakic eyes cataract developed and lens extraction was undertaken within the six month follow-up period.

In 19 eyes in which the retinal break(s) were judged to be securely sealed the silicone oil was removed after a period ranging from 3.5 to 9 weeks (mean 4.5 weeks). In one of these 19 eyes silicone oil was subsequently reinjected because of redetachment.

\section{Results after Six Months (Fig. 1)}

Overall, the retina remained attached at six months in 35 of 39 eyes ( $90 \%)$. 


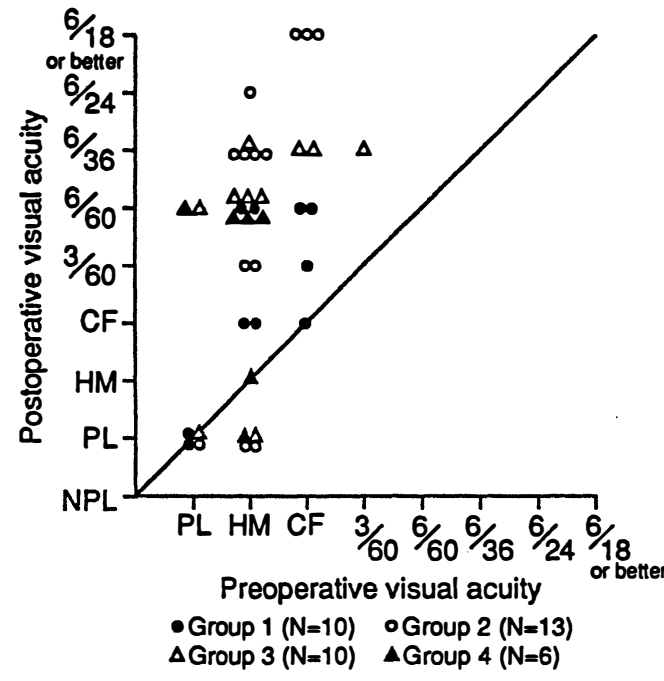

Fig. 1. Scattergram comparing pre and post operative results for 4 groups of patients at 6 months.

Group 1 (Macular breaks) $(\mathrm{n}=10)$

The retina was attached in nine of ten eyes $(90 \%)$ with macular holes; of these nine eyes vision improved in eight, and in four was $6 / 60$ or better. In one eye the retina was detached with marked PVR and the eye eventually became phthisical.

Group 2 (large multiple or posterior breaks) $(\mathrm{n}=13)$

At six months follow-up the retina was reattached in 11 of 13 eyes $(85 \%)$, and of these 11 the vision improved in eight, five with $6 / 36$ or better, and three with $6 / 18$ or better. In two eyes, in spite of further surgical procedures, the retina remained detached as a result of severe PVR.

Group 3 (failed previous surgery without vitrectomy) $(\mathrm{n}=10)$

Following vitrectomy and silicone oil exchange retinal reattachment was achieved in nine eyes $(90 \%)$, and in these the visual acuity improved in eight, four with $6 / 60$ or better, and four with $6 / 36$ or better. In one eye in which there was a choroidal effusion in association with the retinal detachment, small anterior open breaks were identified at the time of surgery by a careful internal search using indentation microsurgery, to allow reattachment with fluid/silicone exchange. In one eye in which the retina was initially reat- tached, detachment occurred within the six months follow-up period as a result of severe PVR.

Group 4 (previous vitrectomy) $(\mathrm{n}=6)$

Retinal reattachment was maintained in all six eyes $(100 \%)$, of which four showed an improvement in vision to $6 / 60$ or better.

\section{Silicone Oil Status}

In 20 eyes silicone oil was left in situ. Eleven were aphakic with a deep anterior chamber present in front of the oil globule, clear cornea and normal intraocular pressure. Of the nine phakic eyes, in two the retina was detached, and in the remaining seven it was felt that the breaks might not be sealed securely.

The general medical condition of four patients in Groups 1 and 3 was such that it was felt that only one further surgical attempt at retinal reattachment could be justified; silicone oil was consequently left in situ in these patients.

\section{Lens Status}

Nineteen eyes were phakic and 20 aphakic, six months after surgery. Of the 20 aphakic eyes 11 were aphakic prior to vitrectomy and fluid/ silicone oil exchange, three underwent lensectomy at the time of surgery, and in six the lens was removed within the six-month follow-up period, because of the development of significant lens opacities.

\section{Complications (Fig. 2)}

\section{Cataract}

Of the 19 eyes which remained phakic at six

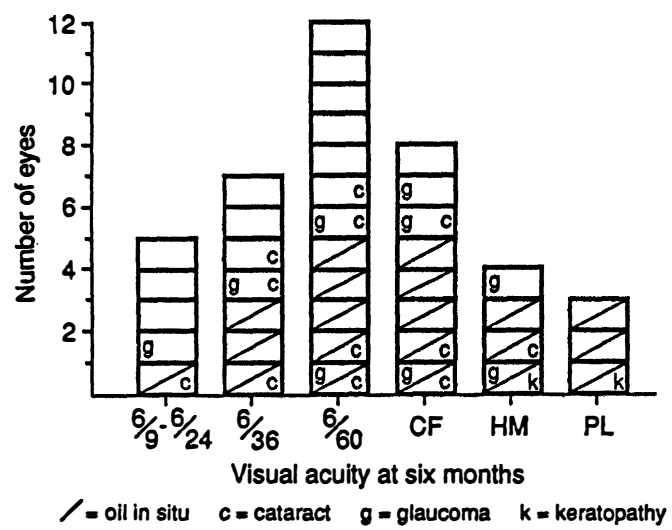

Fig. 2. Histogram showing results and complications at six months for all patients. 
months, nine had silicone oil in situ and 12 (seven with oil in situ and five in which it had been removed) had lens opacities.

\section{Keratopathy}

Corneal endothelial decompensation was present in two eyes $(5 \%)$, in one of which the retina remained detached. This eye had been subjected to previous penetrating trauma with corneal damage.

\section{Glaucoma}

Prior to vitrectomy and fluid/silicone oil exchange two patients required medical treatment for control of intraocular pressure, but at six months follow up, nine eyes needed treatment. In seven (three with oil in situ) the intraocular pressure could be controlled with medical therapy, but two required a drainage tube for satisfactory control.

\section{Discussion}

It is now widely accepted that vitrectomy combined with fluid/silicone oil exchange is a useful technique in the management of complex retinal detachments, although long term complications associated with the use of intraocular silicone oil have until recently restricted its use in all but desperate cases. ${ }^{1-8}$ Recent reports suggest, however, that serious complications can be avoided if silicone oil is removed at an early stage, while the longterm effects associated with its use as a permanent agent for tamponade can be satisfactorily managed. ${ }^{9-10}$

The concept of using silicone oil as an agent for prolonged internal tamponade is an attractive one, for two reasons: firstly, its high viscosity, refractive index (1.34) similar to that of the ocular media and low specific gravity (giving buoyancy to the silicone oil globule in aqueous fluid) facilitate the intraoperative management of highly complicated retinal detachments (e.g. those with giant retinal tears), and secondly because the clarity of view and maintenance of volume enable greater control of events in the post-operative period, without the need for frequent follow up visits and reinjections associated with the use of gases. Furthermore, long term complications associated with use of the more highly insoluble gases may be as serious (or more so) as those encountered with silicone oil, ${ }^{11}$ while other agents such as perfluorocarbon liquids cannot safely be left in the eye for prolonged periods. ${ }^{12}$ For these reasons we have extended our criteria for using silicone oil in the management of complicated retinal detachments to include certain cases in which, although not complicated by advanced PVR, PDR or GRT, the prognosis for long term reattachment and achievement of useful vision is, in our view, less certain if fluid/gas exchange is employed.

It is our practice, in the majority of instances, to manage full thickness macular holes by vitrectomy and fluid/gas exchange with or without endolaser photocoagulation; in a few cases, however, when the outcome is unsuccessful or uncertain, silicone oil exchange can be helpful, e.g. in recurrent retinal detachment with retinal shortening at the posterior pole, where there is insufficient pigmentation for laser uptake, or the patient's general condition is such that strict post-operative posturing is difficult or impossible.

In cases with multiple, large and/or posterior breaks, we normally undertake surgery with or without vitrectomy and fluid/gas exchange, in the first instance. It seems to us, however, that in those instances when retinal detachment occurs, persists or recurs and the development of PVR is encouraged by the presence of large areas of exposed retinal pigment epithelium, intraocular blood or inflammatory exudate, a technique which carries the greatest chance of immediate and sustained retinal reattachment is desirable.

When previous surgery with or without vitrectomy has failed, the conditions favourable to the development of epiretinal membranes are frequently present, while repeated intraocular gas injections can lead to serious complications. Furthermore, patients who have undergone one or more previous operations are not always in such good health physically or mentally that the prospect of further and extensive surgical procedures is acceptable, and the need to return them to their home environment after prolonged and frequent periods of hospitalisation, is pressing.

Past experience suggests that complications from silicone oil are much commoner in aphakic than phakic eyes. ${ }^{13}$ We therefore 
leave the lens in place whenever it is practicable to do so. Only when lens opacities obstruct the operative view, or vitreoretinal surgical pathology in a phakic eye is so anterior that the lens prevents surgical access, do we remove the lens at the time of intraocular silicone oil injection.

Removal of silicone oil is always desirable, not only because of the high risk of complications, notably cataract in phakic eyes and glaucoma and keratopathy in aphakic eyes, but because the possible development of contact retinopathy and optic atrophy, in the long term, cannot be excluded. In this series, we felt sufficiently confident of the security of break closure to remove the silicone oil from $49 \%$ of eyes during the six months follow-up period. It is our policy to remove the silicone oil from phakic eyes with clear lenses at the earliest opportunity, after a minimum three weeks of tamponade, provided that all retinal breaks appear securely sealed. In those phakic eyes which already exhibit some degree of cataract there is less urgency and silicone oil is commonly removed before the cataract becomes so advanced that fundus details are obscured. Extracapsular cataract extraction with intraocular lens can then be undertaken at a later date, when the eye has settled and the retina is seen to remain attached.

In aphakic eyes we remove silicone oil at the first sign of a rise in intraocular pressure or the development of other silicone oil related complications. In some eyes in this series, however, where the break(s) could not be satisfactorily sealed or the integrity of the chorioretinal adhesion was in doubt, for instance those with macular holes overlying bare sclera in the base of staphylomata, silicone oil was left permanently in situ. In such cases, when phakic, we undertook intracapsular cataract extraction (with 6 o'clock iridectomy) when cataract developed, because of the problems engendered by posterior capsular thickening, posterior capsulotomy and silicone oil entering the anterior chamber, after extracapsular cataract extraction in the silicone oil filled eye.

Anatomical failure occurred in four out of 39 cases, in all instances due to the development of severe PVR, a predictable outcome, particularly in retinal detachments with large breaks and failure of previous surgery. Visual recovery in successful cases reflected the effect on macular function of prolonged retinal detachment, the presence of macular breaks or macular pucker. The results of surgery using vitrectomy and fluid/silicone exchange we report here indicate that it is an acceptable method in some cases not complicated by PDR, GRT or, initially, by PVR.

\section{References}

${ }^{1}$ Lean JS, Leaver PK, Cooling RJ, McLeod D: Management of complex retinal detachments by vitrectomy and fluid/silicone exchange. Trans Ophthalmol Soc UK 1982, 102: 203-5.

${ }^{2} \mathrm{Scott} \mathrm{J}$ : Use of liquid silicone in vitrectomised eyes. Dev Ophthalmol 1981, 2: 185-90.

${ }^{3}$ Leaver PK, Cooling RJ, Feretis EB, Lean JS, McLeod D: Vitrectomy and fluid/silicone oil exchange for giant retinal tears: results at six months. Br J Ophthalmol 1984, 68: 432-8.

${ }^{4}$ Grey RHB and Leaver PK: Results of silicone oil injection in massive preretinal retraction. Trans Ophthalmol Soc UK 1977, 97: 238-41.

${ }^{5}$ Mcleod D, Leaver PK, Feretis E: Vitrectomy for severe diabetic eye disease. Trans Ophthalmol Soc UK 1980, 100: 291-8.

${ }^{6}$ Cairns JD and Anand N: Combined vitrectomy, intraocular microsurgery and liquid silicone in the treatment of proliferative vitreo-retinopathy. Aust J Ophthalmol 1984, 12: 239-45.

${ }^{7}$ Leaver PK, Grey RHB, Garner A: Silicone oil injection in the management of massive preretinal retraction. II. Late complications in 93 cases. $\mathrm{Br} J$ Ophthalmol 1979, 63: 361-7.

${ }^{8}$ Haut J, Ullern M, Chermet M, Van Effenterre G: Complications of intraocular injections of silicone combined with vitrectomy. Ophthalmologica 1980, 180: 29-35.

${ }^{9}$ Gonvers M: Temporary silicone oil tamponade in the management of retinal detachment with proliferative vitreoretinopathy. Am J Ophthalmol 1985, 100: 239-45.

${ }^{10}$ Leaver PK, Grey RHB, Garner A: Complications following silicone oil injection. In Streiff ED, ed. Modern Problems in Ophthalmology. Basel: Karger, 1979, 20: 290-4.

${ }^{11}$ Chang S, Lincoff HA, Coleman D, et al. Perfluorocarbon gases in vitreous surgery. Ophthalmology 1985, 92: 651-6.

${ }^{12}$ Chang S, Ozmert E, Zimmerman N: Intraoperative Perfluorocarbon Liquids in the Management of Proliferative Vitreoretinopathy. Am J Ophthalmol 1988, 106: 668-74.

${ }^{13 .}$ Leaver PK and Billington'BM: Vitrectomy and fluid/ silicone-oil exchange for giant retinal tears: 5 years follow-up. Graefe's Arch Clin Exp Ophthalmol 1989, 227: 323-7. 